\title{
Nehmen psychische Störungen zu?
}

\author{
Do Mental Disorders Really Increase?
}

\section{Bibliografie}

DOI 10.1055/s-2008-1067526

Psychiat Prax 2008; 35:

318-320 @ Georg Thieme

Verlag KG Stuttgart · New York .

ISSN 0303-4259

Korrespondenzadressen

Priv.-Doz. Dr. Hermann SpießI

Klinik und Poliklinik für Psychiatrie, Psychosomatik und Psychotherapie der Universität am Bezirksklinikum Regensburg Universitätsstraße 84 93053 Regensburg hermann.spiess|@medbo.de

\section{Priv.-Doz. Dr. Frank Jacobi}

Klinische Psychologie und Psychotherapie, TU Dresden Chemnitzer Straße 46 01187 Dresden jacobi@psychologie.tudresden.de

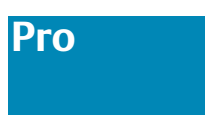

„Psychische und Verhaltensstörungen: Die Epidemie des 21. Jahrhunderts?" [1], so titelte das Deutsche Ärzteblatt den Artikel zum Thema der Zeit am 31.3.2006 und forderte, dass der Erhalt und die Wiederherstellung der seelischen Gesundheit als gesamtgesellschaftliche Aufgabe begriffen werden müsse. Neben den jetzt auch in Fachkreisen wahrgenommenen hohen Prävalenzzahlen für psychische Störungen zeigen insbesondere die Ergebnisse der WHO Global Burden of Disease Study die Bedeutung seelischer Störungen für Gesellschaft und Gesundheitssystem. Zudem lassen Berichte der Krankenkassen bezüglich der Arbeitsunfähigkeit aufhorchen, z.B. habe laut DAK Gesundheitsreport von 2005 [2] die Arbeitsunfähigkeit durch psychische Störungen im Vergleich zu 1997 um 68,7\% zugenommen. Seit 2001 sind psychische Erkrankungen zudem die Hauptursache von Erwerbsunfähigkeit. So werden laut der Deutschen Rentenversicherung jährlich etwa 50000 Menschen wegen einer seelischen Erkrankung frühberentet. Somit stellt sich (wieder) die Frage, ob Inzidenz und/oder Prävalenz psychischer Störungen tatsächlich zunehmen. Die Daten z.B. der Gesundheitsberichterstattung für Deutschland lassen diesbezüglich keine Antwort zu. Wie Richter in seiner aktuellen Übersicht überzeugend darlegt [3], lässt sich anhand von epidemiologischen Daten, die mit ähnlicher Methode an vergleichbaren Stichproben an mindestens 2 Zeitpunkten erhoben wurden, keine Zunahme psychischer Störungen zeigen. Eine generelle Argumentation „pro Zunahme psychischer Störungen“ soll damit hier auch gar nicht erfolgen. Berücksichtigt man aber über die strengen Einschlusskriterien des Reviews von Richter weitere epidemiologische Studien, so finden sich zumindest diskussionswürdige Hinweise auf eine mögliche Zunahme depressiver Störungen. So zeigt zum Beispiel eine große, methodisch hochwertige Bevölkerungsstudie in den USA mit über 42000 Personen eine Zunahme der Prävalenz der Major Depression von 3,3\% (1991/1992) auf 7,1\% (2001/2002) [4]. Aber es bleibt schwierig: Eine im gleichen Zeitraum durchgeführte Studie (NCS und NCS-R) findet dagegen eine Abnahme der Major Depression von 10,1\% (1990-1992) auf 8,7\% (2000-2002) [5,6]; Klassifikationssysteme, Studienpopulation und Fragebögen waren im Vergleich zur Studie von Compton et al. [4] unterschiedlich.

Bezüglich der möglichen Zunahme depressiver Störungen wird u.a. ein sog. Alterskohorteneffekt diskutiert, der sich unabhängig von sozialen und kulturellen Unterschieden zeigt. Das sinkende Ersterkrankungsalter und das erhöhte Erkrankungsrisiko in jüngeren Kohorten lässt sich auch bei Kontrolle möglicher artifizieller Einflussfaktoren nachweisen. Epidemiologische Studien mit jüngeren Kohorten finden höhere Prävalenzen als Studien, die Patienten über 65 Jahre mit einschließen. Zudem zeigt sich eine Zunahme im Schweregrad bei jüngeren Geburtskohorten. Die Betroffenen erkranken häufiger und früher an einer Depression, das Risiko für eine Depression wird pro Dekade verdoppelt - vorausgesetzt, die (retrospektiven) Angaben der Studienteilnehmer sind valide [7]. Hier zeigt sich, dass das Alter beim Interview ein Prädiktor für eine Depression während der Lebensspanne ist. Neben den für den Alterskohorteneffekt möglichen ursächlichen sozialen Faktoren (wie zunehmende Urbanisierung, größere geografische Mobilität, sich rasch verändernde Lebensbedingungen, Veränderungen in der Lebensstruktur, sinkende soziale Unterstützung) und dem Faktor Alter selbst (verringerte emotionale Ansprechbarkeit Älterer für negative Erfahrungen, höhere emotionale Kontrolle negativer Emotionen, psychologische Immunisierung) sind mögliche methodische Artefakte zu bedenken: Neben der vermehrten Wahrnehmung depressiver Symptome bei Jüngeren bzw. der falschen Bewertung bei Älteren werden 
sog. Erinnerungseffekte („Recall Bias“) diskutiert, denn es fällt auf, dass die Lebenszeitprävalenz für Depression nur zweimal höher als die aktuelle Prävalenz ist. Bezieht man andererseits diesen „Recall Bias“ in die Prävalenzberechnung mit ein, müsste bei Männern von einer Lebenszeitprävalenz der Depression von $30 \%$ und bei Frauen von $40 \%$ ausgegangen werden [8]. Damit wären die bisherigen Prävalenzzahlen völlig hinfällig, da viel zu niedrig.

Versorgungspolitisch aktuell relevanter als die Frage der Zunahme der „wahren“ Prävalenz - und auch klarer zu beantworten ist die unzweifelhaft zunehmende Inanspruchnahme medizinischer Leistungen durch Patienten mit psychischen Störungen. Zunehmend mehr psychisch Kranke werden - über alle Diagnosen und Schweregrade hinweg - ambulant und stationär behandelt. Gemäß NCS nahmen die Behandlungsquoten psychischer Störungen im Vergleich von 1990/1992 zu 2001 / 2002 um über $10 \% \mathrm{zu}$ [9]. Dies ist eine an sich erfreuliche Entwicklung angesichts der bekanntermaßen niedrigen sog. Behandlungsprävalenz [10]. So haben nur etwa 50\% der Patienten mit Depression eine professionelle Behandlung aufgesucht oder erhalten. Obgleich sich im Vergleich zu den 90er-Jahren die Versorgungssituation im primärärztlichen Bereich verbessert hat, ist der Anteil nicht erkannter bzw. unbehandelter Patienten noch immer zu hoch, die Überweisungsrate in den psychiatrisch-psychotherapeutischen Fachbereich und die Verordnung von Antidepressiva und Psychotherapie zu niedrig. Dennoch findet sich auch in der stationär-psychiatrischen Behandlung ein seit Jahren zunehmender Anteil von Patienten mit affektiven Störungen.

Aufgrund der erheblichen gesundheitsökonomischen Implikationen psychischer und gerade depressiver Störungen sollten zukünftig neben regelmäßigen Bevölkerungssurveys und prospektiven Kohortenstudien auch routinemäßig erhobene Daten der ambulanten und stationären psychiatrisch-psychotherapeutischen Versorgung (z.B. DGPPN-BADO und Krankenkassendaten) systematisch analysiert werden, um Morbiditätsverschiebungen genauer evaluieren zu können, um - neben der Frage der Zunahme psychischer Störungen - auch frühzeitig und gezielt Präventionsmaßnahmen einleiten zu können [11]

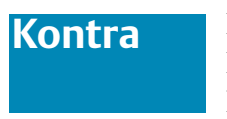

Nein, psychische Störungen nehmen nicht zu. Psychische Störungen sind zwar ausgesprochen häufig und kostenträchtig $[12,13]$ und werden in ihrer Bedeutung heute zunehmend wahrgenommen und gewürdigt - die Erklärung für die drastischen Zunahmen in Krankenkassenstatistiken liegt aber nicht in einer Erhöhung der allgemeinen Prävalenz psychischer Störungen.

Es entbehrt zwar nicht einer gewissen Plausibilität, dass die verstärkte Wahrnehmung und die gestiegene gesundheitspolitische Relevanz psychischer Störungen auf einer tatsächlichen Zunahme der Erkrankungsfälle beruhen könnte, die wiederum mit einer Zunahme der Risikofaktoren in Verbindung steht. In der Tat können soziologische Veränderungen in den vergangenen Jahrzehnten hinsichtlich anerkannter Risiko- und Schutzfaktoren festgestellt werden. Inwiefern solche gesellschaftlichen Veränderungen tatsächlich mit veränderten Raten psychischer Störungen einhergehen, bleibt allerdings Spekulation, solange die entsprechenden Prävalenzen nicht in derselben Population mit derselben Methodik in größeren Zeitabständen wiederholt gemessen werden. Die wenigen Studien, in denen psychische Störungen über längere Zeiträume vergleichbar erfasst wurden, legen jedoch den Schluss nahe, dass es insgesamt keine bedeutsame
Zunahme gab. In großen Studien in den USA $[5,14]$ und Großbritannien [15] fanden sich 10 Jahre nach der ersten Erhebung Anfang der 90er-Jahre bei einer Wiederholungsmessung kaum Prävalenzunterschiede, sodass dort zumindest eine dramatische Zunahme unwahrscheinlich ist. Auch entsprechende Übersichten im Kinder- und Jugendbereich kommen zu dem Schluss, dass psychische Störungen zumindest in ihrer Gesamtheit keinesfalls dramatisch zugenommen haben $[16,17]$. Ebenso fanden sich keine entsprechenden Hinweise in einer der umfassendsten je durchgeführten Längsschnittstudien [18]. Richter et al. [3] fassen in ihrer Übersichtsarbeit derartige Studien mit der validesten Methodik - der wiederholten Messung in denselben repräsentativen Populationen mit derselben Methodik (Störungskriterien, Erhebungsmethoden, Stichprobendesign) zusammen und kommen zu einem zwar nicht eindeutigen, aber doch sehr deutlichen Befund: Möglicherweise gab es einen Anstieg der Prävalenz und Inzidenz psychischer Störungen in den ersten Dekaden nach dem Zweiten Weltkrieg; dieser Trend hat sich jedoch offenbar nicht weiter fortgesetzt. Auch wenn gewisse Fluktuationen festgestellt werden können und die Einschlusskriterien für die in diesem Review zitierten 41 Studien streng waren, kann damit behauptet werden: Zumindest auf Bevölkerungsebene ist die Prävalenz psychischer Störungen trotz gesellschaftlicher Veränderungen nicht besonders volatil!

Und es mag auch einen Bias dahingehend geben, dass jüngere Ereignisse und Veränderungen als besonders belastend oder zumindest ganz außerordentlich und damit irritierend empfunden werden wie folgende (hypothetische) Aussagen verdeutlichen sollen:

- 2008: „Noch nie gab es Umwälzungen wie in den letzten 20 Jahren (mit der Globalisierung zusammenhängende Verwerfungen am Arbeitsmarkt, Finanzkrisen, Terrorismus, Wiedervereinigung, Europäische Union etc.) ...“

- 1988: „Noch nie gab es Umwälzungen wie in den letzten 20 Jahren (Wertewandel im Zuge von 1968, kalter Krieg mit Aufrüstung und Kriegsangst, Terrorismus, Umweltzerstörung, aber auch Gewinn an Emanzipation und Freiheitsgraden etc.) ..."

- 1968: „Noch nie gab es Umwälzungen wie in den letzten 20 Jahren (Aufbauarbeit und Notstand der Nachkriegszeit, Atomwaffen und Kalter Krieg, Vielzahl weiterer Kriege, Werteverfall, (Nicht-)Auseinandersetzung mit Nazi-Vergangenheit, aber auch Gewinn an Wohlstand etc.).

Man möchte sagen: Das muss die Seelen der jeweiligen Kohorten doch erschüttert haben! Gewiss verändern sich gesellschaftliche Befindlichkeiten. Stressfaktoren und Unsicherheiten sind stets im Wandel begriffen, aber auch Anpassungsprozesse und neue Schutzfaktoren dürften im Hinblick auf psychische Störungen in ihrer Allgemeinheit und in der Allgemeinbevölkerung von Bedeutung sein. Und jede Zeit hat ihre vulnerablen Individuen, die auf Stressoren mit psychischen Störungen (erkannt oder unerkannt) reagieren. Somit sollten Befunde dahingehend, dass in jüngeren Kohorten psychische Störungen bzw. deren Lebenszeitrisiko drastisch zunehmen, nicht nur aufgrund methodischer Argumente relativiert werden $[3,19]$. Auch sollten andere Indikatoren psychischer Gesundheit in der Bevölkerung nicht aus dem Auge verloren werden: Suizidraten sinken in den letzten Dekaden kontinuierlich, Alkohol- und Nikotinkonsum sind im Schnitt gesunken, und die gemessene gesamtgesellschaftliche Lebensqualität bleibt erstaunlich unabhängig von gesellschaftlicher Wohlfahrt und Wandel weitgehend gleich [20]. 
Abschließend kann die Frage nach einer (Nicht-)Zunahme psychischer Störungen allerdings noch nicht geklärt werden. Erstens gibt es Ausnahmen, die zumindest in einzelnen Störungsbereichen deutliche Zuwächse in einem 10-Jahres-Zeitraum gefunden haben [4]. Zweitens gibt es Störungsbereiche, deren Zunahme aufgrund demografischer Veränderungen gesichert ist (Demenzen) bzw. hoch wahrscheinlich ist (Depressionen und Suizid im hohen Lebensalter). Bei Substanzabhängigkeit und -missbrauch haben wir es ferner mit zeitgeistabhängigen Zyklen und Ungleichzeitigkeiten in verschiedenen Kohorten zu tun. Manche Bevölkerungsgruppen sind insgesamt hinsichtlich psychischer Störungen unzureichend untersucht (z.B. Hochbetagte, Migranten). Zudem ist unklar, ob der Einbezug weiterer Störungskategorien (z.B. Burn-out, nicht stoffgebundene Süchte) bzw. eine Reaktivierung früherer diagnostischer Kategorien (z. B. Neurasthenie) in entsprechenden Schätzungen eine Zunahme belegen würde.

Doch trotz solcher Ausnahmen und Wissenslücken macht es keinen Sinn, bei psychischen und Verhaltensstörungen von einer „Epidemie des 21. Jahrhunderts“ oder einem „Age of Depression“ zu sprechen. Andere Aspekte als eine Epidemiehypothese sind heute eigentlich von Belang, z.B. ob und wie die Versorgungssituation angesichts der klaren früheren Unterschätzung psychischer Störungen dem tatsächlichen Bedarf angepasst werden kann [21]. Um präzise Aussagen über den Wandel psychischer Symptomatik machen zu können, sind Verlaufsstudien und wiederholte Querschnittsstudien in denselben Populationen notwendig. Aus gesundheitswissenschaftlicher Perspektive müssen zudem auch der Schweregrad der Störung (Symptombelastung, Beeinträchtigungsgrad, Chronifizierung) und der dadurch moderierte Behandlungsbedarf in die Analyse einbezogen werden. In Deutschland wäre hier übrigens eine erneute Untersuchung psychischer Störungen in der Bevölkerung im kommenden Bundesgesundheitssurvey (einschließlich der 10-jährigen längsschnittlichen Komponente) hoch aufschlussreich.

Keine Gesundheit ohne psychische Gesundheit [22] ist gerade im derzeitigen „Jahrzehnt des Verhaltens“ eine zentrale Botschaft, die nicht durch drastische Überzeichnungen aufgrund einer „gefühlten Zunahme“ psychischer Störungen verstärkt werden muss. Auch ohne eine Zunahme psychischer Störungen ist Handlungsbedarf unbedingt gegeben!

\section{Literatur}

1 Weber A, Hörmann G, Köllner V. Psychische und Verhaltensstörungen: Die Epidemie des 21. Jahrhunderts? Dt Ärztebl 2006; 103: A 834-841

2 DAK Versorgungsmanagement. DAK Gesundheitsreport 2005. 2005; online: http://www.sozialpolitik-aktuell.de/docs/DAK-Gesundheits report_2005.pdf [accessed 3.1.2008]

3 Richter D, Berger K, Reker T. Nehmen psychische Störungen zu? Eine systematische Literaturübersicht. Psychiat Prax 2008; 35: 321-330
4 Compton WM, Conway KP, Stinson FS et al. Changes in the prevalence of major depression and comorbid substance use disorders in the United States between 1991-1992 and 2001-2002. Am J Psychiatry 2006; 163: 2141-2147

5 Kessler RC, McGonagle KA, Zhao S et al. Lifetime and 12-month prevalence of DSM-III-R psychiatric disorders in the United States: results from the National Comorbidity Survey. Arch Gen Psychiatry 1994; 51: 8-19

6 Greenberg PE, Kessler RC, Birnbaum HG et al. The economic burden of depression in the United States: how did it change between 1990 and 2002? J Clin Psychiatry 2003; 64: 1465-1475

7 Simon GE, Vonkorff M, Ustun TB et al. Is the lifetime risk of depression actually increasing? J Clin Epidemiol 1995; 48: 1109-1118

8 Kruijshaar ME, Barendregt J, Vos T et al. Lifetime prevalence estimates of major depression: An indirect estimation method and a quantification of recall bias. Eur J Epidemiol 2005; 20: 103-111

9 Kessler RC, Demler O, Frank RG et al. Prevalence and treatment of mental disorders, 1990 to 2003. N Engl J Med 2005; 352: 2515-2523

10 Jacobi F, Wittchen HU. „Diagnoseträger“ und Patienten: Zur Epidemiologie behandelter und unbehandelter psychischer Störungen in Deutschland. In: Kosfelder J, Michalak J, Vocks S, Willutzki U, Hrsg: Fortschritte der Psychotherapieforschung. Göttingen: Hogrefe, 2005: 25-36

11 Spießl H, Hübner-Liebermann B, Hajak G. Depression - und viele schauen (noch) weg! Psychiat Prax 2007; 33: 53-54

12 Wittchen HU, Jacobi F. Size and burden of mental disorders in Europe a critical review and appraisal of 27 studies. Eur Neuropsychopharmacol 2005; 15: 357-376

13 Gunther $\mathrm{OH}$, Friemel S, Bernert $S$ et al. The burden of depressive disorders in Germany - Results from the European study of the epidemiology of mental disorders (ESEMeD). Psychiat Prax 2007; 34: 292-301

14 Kessler RC, Chiu WT, Demler 0 et al. Prevalence, severity, and comorbidity of 12-month DSM-IV disorders in the national comorbidity survey replication. Arch Gen Psychiatry 2005; 62: 617-627

15 Singleton N, Bumpstead R, O'Brien M et al. Psychiatric morbidity among adults living in private households. National Statistics 154. London: The Stationery Office, 2001

16 Costello EJ, Erkanli A, Angold A. Is there an epidemic of child or adolescent depression? J Child Psychol Psychiatry 2006; 47: 1263-1271

17 Ihle W, Esser G. Epidemiologie psychischer Störungen im Kindes- und Jugendalter: Prävalenz, Verlauf, Komorbidität und Geschlechtsunterschiede. Psychol Rundsch 2002; 53: 159-169

18 Murphy JM, Laird NM, Monson RR et al. A 40-year perspective on the prevalence of depression: the Stirling County Study. Arch Gen Psychiatry 2000; 57: 209-215

19 Mauz E, Jacobi F. Psychische Störungen und soziale Ungleichheit im Geburtskohortenvergleich. Psychiat Prax 2008; 35: 343-352

20 Kovess $V$. The state of mental health in the European Union. European Commission. 2006; online: http://ec.europa.eu/health/ph_projects/ 2001/monitoring/fp_monitoring_2001_frep_06_en.pdf [accessed 1.11.2007]

21 Jacobi F, Hoyer J. Gesundheitsberichterstattung über Psychotherapie nach dem Gesundheitsmodernisierungsgesetz: Ein kritischer Fall. Psychotherapeutenjournal 2008; 2: 140-143

22 European Commission. Green Paper. Improving the mental health of the population: Towards a strategy on mental health for the European Union. 2005; online: http://ec.europa.eu/health/ph_determinants/ life_style/mental/green_paper/mental_gp_en.pdf [accessed 1.4.2008] 